\title{
Rotation Effects in Relativity
}

\author{
Matteo Luca Ruggiero \\ Department of Applied Science and Technology, Politecnico di Torino, 10129 Torino, Italy; matteo.ruggiero@polito.it \\ Received: 10 November 2020; Accepted: 25 November 2020; Published: 27 November 2020

Rotation has always been a central thread in physics and has influenced its development. In the Aristotelian universe, the planets and their spheres were made of a first element different from earth, air, fire, and water-the terrestrial elements-and this element could only move in circles unlike the other ones, which could only move up and down. Hence, in the heavenly spheres only circular motion was permitted. Aristotle's thought survived until the Renaissance, where the Copernican revolution completely changed our view of the universe; the transition from geocentrism to heliocentrism was also determined by Kepler: with his laws of planetary motion he provided a beautiful and simple way of understanding the meticulous astronomical observations collected by Tycho Brahe and many others. According to Kepler's laws, planets rotate around the Sun along ellipses: it took the genius of Newton (who stood "on the shoulders of Giants") to show that an inverse square law of gravity and the principles of dynamics accounted for the elliptical orbits and the other laws of planetary motion. Hence, the need to explain the rotation of planets around the Sun stimulated the birth of classical mechanics. The paradigm of Newtonian physics was shaken by Einstein, two hundred years later: in order to reconcile the principle of relativity with electromagnetism and to explain the constancy of the speed of light, space and time (which in Newtonian physics are absolute entities independent of physical events) lost their individuality and joined together to form the four-dimensional spacetime continuum. Rotation had an important role also in the making of the theory of general relativity: before obtaining his definitive equations for the gravitational field, in 1915 Einstein showed that his theory was able to explain the precession of the perihelion of Mercury, discovered 70 years before by Le Verrier; Einstein was deeply motivated by this important result, which suggested that he was on the right track towards a relativistic theory of gravity [1]. Today, understanding the rotation curves of galaxies is one of the most challenging problems in astrophysics, because the observations are not in agreement with the current gravity paradigm and, in order to explain them, the existence of dark matter is required [2,3].

Everything seems to rotate in our universe: the peculiarities of rotating reference frames are recognised both in Newtonian physics, where the Foucault's pendulum provided a spectacular evidence of the absolute character of rotation and, also, in relativistic physics, where the Sagnac effect stimulated a long and interesting debate on the foundations of relativity (see e.g., the monograph edited by Rizzi and Ruggiero [4]). The purpose of this Special Issue is to focus on what we know about the effects of rotation in relativistic theories of gravity, which include not only Einstein's special and general theories, but also the theories that have been proposed to solve the difficulties deriving from observations at very large scales (i.e., the problems of dark matter and dark energy) and, also, to reconcile gravity with a quantum description. Rotation effects are quite ubiquitous, since they are important to understand the foundations of relativity, are relevant to describe and understand astrophysical phenomena, are significant to test the theoretical predictions by experiments or observations, can be important to evaluate the impact of extended theories of gravity and, also, could be a key to comprehend the interplay between gravity and quantum mechanics. The papers collected in this Special Issue encompass all these main topics. 
It is well known that Mach's ideas influenced the development of Einstein's theory of gravitation, even if there are difficulties in incorporating Mach's principle in general relativity [5]. This theme is resumed by Putz [6], who presents here a non-relativistic theory of inertia based on Mach principle. The role of simultaneity in rotating frames is important not only for the principles of relativity, but it is also relevant for the current timing standard which allows the working of the Global Positioning System. Simultaneity in the context of relativistic rotation is focused on in the paper by Koks [7]. The measurement process in rotating reference frame is relevant to understand the experimental results: a careful analysis of the transformation of electromagnetic fields from inertial to rotating frames is carried out by Speake and Ortolan [8]. We are in the era of multi-messenger astronomy: a given astrophysical source can be detected by means of different messengers, such as photons, neutrinos and, eventually, gravitational waves. Few years ago, the first direct detection of gravitational waves [9] constituted the birth of gravitational waves astronomy. Rotation effects could be relevant to design new type of gravitational waves detectors, based on the gravitomagnetic resonance, as suggested by Ruggiero and Ortolan [10]. Here, the link between gravitational waves, vorticity (which is strictly related to the rotation features of the congruence of observers) and the super-energy flux is analysed by Herrera [11].

Rotation effects are important in several astrophysical phenomena. In particular, Semerák and Čížek [12] focused on the first-order perturbation of a Schwarzschild black-hole space-time, determined by a disk rotating around the hole. Accretion disks rotating around black holes are discussed in the paper by Stuchlík et al. [13] in connection with the cosmic repulsion and the magnetic fields acting upon them. Relativistic rotation in neutron star is analysed by Pétri [14]: in particular, the rotation effects can be probed by the radiation produced by the neutron star magnetospheres, and this could help to better understand particle electrodynamics in such extreme conditions. The Gödel model of rotating Universe [15] is important for its implications on closed time-like curves, causality and the meaning of time, even though its predictions are not in agreement with observational constraints. The problem of global rotation in general relativity is analyzed in the contribution by Korotky et al. [16]: they show that there are cosmological models with rotation and expansion which give a natural explanation of the modern values of the acceleration parameter without requiring the existence of dark matter and dark energy.

The continuous technological improvements will certainly allow further tests of gravity, both in space and, hopefully, on the Earth. In particular, Iorio [17] describes HERO (Highly Eccentric Relativity Orbiter), a space-based mission concept aimed to perform several tests of post-Newtonian gravity, among which the Lense-Thirring effect, deriving from the rotation of the source of the gravitational field. Acedo [18] focuses on another mission concept, called CHRONOS, to study orbital resonances around Jupiter. Eventually, Schreiber et al. [19] reviews the large ring laser gyroscopes, which can be used to measure general relativistic precessions [20].

As we have said before, large scale observations of the Universe can be explained in the framework of Einstein's theory requiring the existence of dark matter and dark energy, which need to have peculiar characteristics that have not been observed in the fundamental particles that we know. Accordingly, there are various attempts to modify general relativity to explain the observations in a natural way and without requiring the existence of these dark entities. In these modified theories rotations effects are relevant. Both special and general relativity are based on the locality hypothesis and, accordingly, an accelerated observer is assumed to be physically identical with a hypothetical inertial observer with the same position and velocity; nonlocal gravity is a classical nonlocal generalisation of Einstein theory of gravitation based on an analogy with the nonlocal electrodynamics of media. This model could be useful to simulate the effects of dark matter without requiring the existence of unknown fluids. Here, Mashhoon and Hehl [21] study the stationary gravitational field of an isolated rotating astronomical source, in linear approximation, and compare their results with the standard general relativistic approach. General relativity predicts the existence of a gravitomagnetic field, produced by mass currents, in analogy to what happens 
for the magnetic field, produced by charge currents: more generally, it is possible to describe gravitational effects on the basis of a gravitoelectrogmagnetic analogy [22,23]. Gravitomagnetic effects, in the framework of Teleparallel Gravity, are explored by Farrugia et al. [24], in connection with recent and future missions. Dymnikova and Galaktionov [25] consider a model of nonlinear electrodynamics minimally coupled to gravity and study the dynamics of electromagnetic fields of rotating electrically charged black holes. Hess and López-Moreno [26] focus on another model of modified gravity, the so-called pseudo-complex General Relativity and, in this context, they study Kerr black hole in presence of vacuum fluctuations that are determined by the modified gravity model.

One of the most challenging problems in theoretical physics is the relation between general relativity and quantum mechanics: in fact, up today, we do not know how to reconcile the standard model of particle physics with the geometrical description of gravity. In this context, spin and spinning particles could have an important role. In fact, while spin is a fundamentally quantum property, in general relativity spin is present only at classical level deriving from the rotation of finite-size bodies. O'Connell [27] focuses on the role of spin in special relativity and discusses the Pryce-Newton-Wigner position operators which are important for the treatment of classical relativistic particles in general relativity. The behaviour of two-level quantum systems in the gravitational field of rotating and non rotating sources is studied by Papini [28]. Eventually, Lake et al. [29] derive generalised uncertainty relations for orbital angular momentum and spin in the smeared-space model of quantum geometry.

In conclusion, I would like to thank all colleagues who contributed to this Special Issue: it was a privilege to have the possibility of collaborating with them and to share their enthusiasm in the achievement of this project. I hope that this collection of papers can be an important reference for the researchers in these fields. Moreover, I would like to thank the Universe Editorial office for the continuous and active support.

Funding: This research received no external funding.

Conflicts of Interest: The author declares no conflict of interest.

\section{References}

1. Pais, A. Subtle is the Lord: The Science and the Life of Albert Einstein; Oxford University Press: New York, NY, USA, 1982.

2. Rubin, V.C.; Ford, W.K., Jr.; Thonnard, N. Extended rotation curves of high-luminosity spiral galaxies. IV-Systematic dynamical properties, SA through SC. Astrophys. J. 1978, 225, L107-L111. [CrossRef]

3. Binney, J.; Tremaine, S. Galactic Dynamics; Princeton University Press: Princeton, NJ, USA, 2011.

4. Rizzi, G.; Ruggiero, M.L. Relativity in Rotating Frames: Relativistic Physics in Rotating Reference Frames; Springer Science \& Business Media: Berlin/Heidelberg, Germany, 2013; Volume 135.

5. Brans, C.; Dicke, R.H. Mach's principle and a relativistic theory of gravitation. Phys. Rev. 1961, 124, 925. [CrossRef]

6. Putz, V. A Theory of Inertia Based on Mach's Principle. Universe 2019, 5, 188. [CrossRef]

7. Koks, D. Simultaneity and Precise Time in Rotation. Universe 2019, 5, 226. [CrossRef]

8. Speake, C.C.; Ortolan, A. Measuring Electromagnetic Fields in Rotating Frames of Reference. Universe 2020, 6, 31. [CrossRef]

9. Abbott, B.P.; Abbott, R.; Abbott, T.; Abernathy, M.; Acernese, F.; Ackley, K.; Adams, C.; Adams, T.; Addesso, P.; Adhikari, R.; et al. Observation of gravitational waves from a binary black hole merger. Phys. Rev. Lett. 2016, 116, 061102. [CrossRef]

10. Ruggiero, M.L.; Ortolan, A. Gravito-magnetic resonance in the field of a gravitational wave. Phys. Rev. D 2020, 102, 101501. [CrossRef]

11. Herrera, L. Gravitational Radiation, Vorticity And Super-Energy: A Conspicuous Threesome. Universe 2019, 5, 164. [CrossRef] 
12. Semerák, O.; Čížek, P. Rotating Disc around a Schwarzschild Black Hole. Universe 2020, 6, 27. [CrossRef]

13. Stuchlík, Z.; Kološ, M.; Kovář, J.; Slaný, P.; Tursunov, A. Influence of Cosmic Repulsion and Magnetic Fields on Accretion Disks Rotating around Kerr Black Holes. Universe 2020, 6, 26. [CrossRef]

14. Pétri, J. Electrodynamics and Radiation from Rotating Neutron Star Magnetospheres. Universe 2020, 6, 15. [CrossRef]

15. Gödel, K. An Example of a New Type of Cosmological Solutions of Einstein's Field Equations of Gravitation. Rev. Mod. Phys. 1949, 21, 447-450. [CrossRef]

16. Korotky, V.A.; Masár, E.; Obukhov, Y.N. In the Quest for Cosmic Rotation. Universe 2020, 6, 14. [CrossRef]

17. Iorio, L. A HERO for General Relativity. Universe 2019, 5, 165. [CrossRef]

18. Acedo, L. Monitoring Jovian Orbital Resonances of a Spacecraft: Classical and Relativistic Effects. Universe 2019, 5, 222. [CrossRef]

19. Schreiber, K.U.; Gebauer, A.; Kodet, J.; Anyi, C.L.; Wells, J.P.R. Rotation Sensing Lasers in General Relativity: Some Technical Notes and Current Advances. Universe 2019, 5, 190. [CrossRef]

20. Bosi, F.; Cella, G.; Di Virgilio, A.; Ortolan, A.; Porzio, A.; Solimeno, S.; Cerdonio, M.; Zendri, J.P.; Allegrini, M.; Belfi, J.; et al. Measuring gravitomagnetic effects by a multi-ring-laser gyroscope. Phys. Rev. D 2011, 84, 122002. [CrossRef]

21. Mashhoon, B.; Hehl, F.W. Nonlocal Gravitomagnetism. Universe 2019, 5, 195. [CrossRef]

22. Ruggiero, M.L.; Tartaglia, A. Gravitomagnetic effects. Nuovo Cim. 2002, B117, 743-768.

23. Mashhoon, B. Gravitoelectromagnetism: A Brief review. arXiv 2003, arXiv:gr-qc/0311030.

24. Farrugia, G.; Said, J.L.; Finch, A. Gravitoelectromagnetism, Solar System Tests, and Weak-Field Solutions in $\mathrm{f}$ (T,B) Gravity with Observational Constraints. Universe 2020, 6, 34. [CrossRef]

25. Dymnikova, I.; Galaktionov, E. Dynamics of Electromagnetic Fields and Structure of Regular Rotating Electrically Charged Black Holes and Solitons in Nonlinear Electrodynamics Minimally Coupled to Gravity. Universe 2019, 5, 205. [CrossRef]

26. Hess, P.O.; López-Moreno, E. Kerr Black Holes within a Modified Theory of Gravity. Universe 2019, 5, 191. [CrossRef]

27. O'Connell, R.F. Rotation and Spin and Position Operators in Relativistic Gravity and Quantum Electrodynamics. Universe 2020, 6, 24. [CrossRef]

28. Papini, G. Gravitational Qubits. Universe 2019, 5, 123. [CrossRef]

29. Lake, M.J.; Miller, M.; Liang, S.D. Generalised Uncertainty Relations for Angular Momentum and Spin in Quantum Geometry. Universe 2020, 6, 56. [CrossRef]

Publisher's Note: MDPI stays neutral with regard to jurisdictional claims in published maps and institutional affiliations.

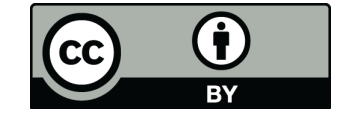

(C) 2020 by the authors. Licensee MDPI, Basel, Switzerland. This article is an open access article distributed under the terms and conditions of the Creative Commons Attribution (CC BY) license (http:/ / creativecommons.org/licenses/by/4.0/). 